\title{
Análise de paclobrutrazol em solos de áreas cultivadas com diferentes variedades de mangueira no Vale do São Francisco empregando QuEcHers e CLAE
}

\author{
Analysis of paclobutrazol in soil from different types of mango in the São Francisco \\ Valley using Quechers and HPLC
}

\author{
P. T. S. Silva ${ }^{1 *}$; L. S. S. Sousa ${ }^{2}$; C. R. S. Neta ${ }^{2}$; M. A. Mouco'; W. L. Simões ${ }^{1}$; A. \\ V. Ferraz ${ }^{3}$ \\ ${ }^{1}$ Embrapa Semiárido, 56302-970 Petrolina-PE, Brasil \\ ${ }^{2}$ Instituto Federal do Sertão de Pernambuco, 56302-900 Petrolina-PE, Brasil, \\ ${ }^{3}$ Universidade Federal do Vale do São Francisco, 56304-917 Petrolina-PE, Brasil
}

*paula.silva@embrapa.br

(Recebido em 13 de julho de 2017; aceito em 19 de setembro de 2017)

\begin{abstract}
O paclobutrazol (PBZ) é um composto do grupo dos triazois, muito usado na agricultura como regulador de crescimento. É aplicado geralmente no solo, onde pode permanecer ativo por alguns anos. No Vale do submédio do São Francisco, esse composto vem sendo aplicado para garantir a produção de manga durante todo o ano. O objetivo desse estudo foi validar um método para quantificar o paclobutrazol no solo, avaliando áreas cultivadas com mangueira no Vale do São Francisco. A importância desse estudo é devido a frequencia do uso do PBZ aplicado no solo, que poderá acarretar risco de contaminação ambiental e também da possibilidade da redução da dosagem aplicada, para melhorar a eficiência da produção da mangueira. Para isso, foram coletadas amostras de solo em fazendas localizadas no município de Petrolina-PE e Juazeiro-BA. O PBZ foi extraído por QuEchers e analisado por cromatografia liquida de alta eficiência (CLAE). Resíduos do PBZ foram encontrados em amostras de solo sob diferentes variedades de mangueira $\left(0,18-6,86 \mathrm{mg} \mathrm{kg}^{-1}\right)$. Os resultados encontrados demonstraram que a extração por QuEchErs e a determinação por CLAE foi eficiente para determinação do PBZ no solo.

Palavras-chave: método de validação; regulador de crescimento; soil; QuEchErs; CLAE
\end{abstract}

Paclobutrazol (PBZ) is a compound from the triazole group that is often used as an agricultural growth regulator. It is usually applied to the soil, where it can remain active for a number of years. In the São Francisco valley, this compound has been used to ensure the year-round production of mangoes. The aim of the present study was to develop a method of quantifying Paclobutrazol in soil samples from different areas with different types of mango. This research is important due to the widespread application of PBZ in soils, which can involve a risk of environmental contamination. The results could lead to reductions in its use, thereby reducing the costs of mango production. Soil samples were collected from farms in the municipality of Petrolina-PE and Juazeiro-BA. PBZ was extracted using QuEchers and analyzed based on liquid chromatography. PBZ residue was found in soil samples taken from different types of mango (0,18-6,86 mg $\mathrm{kg}^{-1}$ ), especially the Kent variety. The results confirmed that the method was effective in terms of determining the quantity of PBZ in the soil

Keywords: Method validation; growth regulator; soil; QuEchErs; CLAE

\section{INTRODUÇÃO}

A mangueira (Mangifera indica L.), originária da Índia, é uma espécie frutífera da família Anacardiaceae. O seu fruto, a manga, nos últimos 15 anos tornou-se uma das frutas mais procuradas por consumidores brasileiros e internacionais, especialmente depois que as ações de melhoramento genético resultaram em espécies não fibrosas, mais macias e saborosas [1]. Segundo o Anuário Brasileiro da Fruticultura [2], a manga foi a principal fruta exportada pelo Brasil em receita gerada 
em 2015, destacando-se o crescimento de área plantada desta cultura no Vale do São Francisco, na região de Juazeiro (BA) e Petrolina (PE).

Para o eficiente manejo de produção da mangueira, de forma a atender os diferentes mercados disponíveis, em períodos adequados à comercialização, é necessário o uso de substâncias reguladoras de crescimento vegetal, que possibilitam modificar os processos que estimulam o florescimento [3]. A substância reguladora utilizada, preferencialmente, no cultivo da mangueira é o paclobutrazol (PBZ), um derivado dos triazóis que apresenta uma estrutura química com fórmula molecular $\left(\mathrm{C}_{15} \mathrm{H}_{20} \mathrm{ClN}_{3} \mathrm{O}\right)$ e massa molecular $293,8 \mathrm{~g} \mathrm{~mol}^{-1}$. Os triazóis formam um grupo de reguladores vegetais que inibem a síntese das giberelinas [4]. As giberelinas integram o grupo dos hormônios vegetais, que são compostos orgânicos, de ocorrência natural, produzido na planta, o qual a baixa concentração promovem, inibem ou modificam processos morfológicos e fisiológicos do vegetal e tem consistentemente mostrado a habilidade para afetar o processo de desenvolvimento. $\mathrm{O}$ desenvolvimento e crescimento das plantas são regulados pela interação entre hormônios, incluindo as giberelinas. Na regulação da floração da mangueira e de outras culturas frutíferas, as giberelinas parecem ser os hormônios mais ativos. Altos níveis de GA inibem a floração e estimulam o crescimento vegetativo e o seu declínio promoverá a floração. O papel principal da aplicação dos reguladores vegetais é a supressão da biossíntese de giberelina [5].

Neste contexto, o PBZ é utilizado para induzir o repouso dos ramos por três a quatro meses, permitindo o acúmulo de reservas e a diferenciação das gemas, resultando no processo de indução floral da mangueira [6].

Apesar da sua eficiência no manejo da floração e produção da mangueira, em virtude do PBZ ser aplicado via solo, devido a maior eficiência no movimento acrópeto na planta, apresenta como inconveniente uma elevada persistência na planta e no solo [7].

Essa persistência no solo poderá acarretar a contaminação dos corpos d'água que estejam próximo e também as águas subterrâneas, podendo se tornar tóxico ao homem e aos animais [8]. Além disso, essa persistência do PBZ pode afetar também severamente o crescimento e desenvolvimento de safras seguintes nos cultivos perenes $[9,10]$. Por isso, existe a necessidade de monitoramento contínuo das concentrações do composto no solo [11,12].

Para realização desse acompanhamento, o desenvolvimento de métodos analíticos de extração e análise são importantes. Nos últimos anos, métodos de preparo de amostra baseados na minimização do uso de solventes orgânicos e mais rápido têm sido desenvolvidos, merecendo destaque o QuEChERS (Quick, Easy, Cheap, Effective, Robust and Safe). Esse método baseia-se no preparo da amostra nas seguintes etapas: extração com acetonitrila, seguida da partição, promovida pela adição de sais (ex. sulfato de magnésio, $\mathrm{MgSO}_{4}$ e cloreto de sódio, $\mathrm{NaCl}$ ). Um novo método de limpeza denominado Extração em Fase Sólida Dispersiva (Dispersive Solid Phase Extraction, D-SPE) foi proposto juntamente com o método QuEChERS [13].

O método QuEChERS tem como vantagens ser rápido, fácil, econômico, efetivo, robusto e seguro e vem sendo muito empregado na extração de pesticidas em frutas [14]. Em relação a extração de pesticidas em solo, alguns trabalhos foram desenvolvidos mostrando eficiência desse método para extração de contaminantes em solo $[15,16]$. A praticidade e os excelentes resultados fornecidos pelo método QuEChERS permite explorar as possibilidades oferecidas pela instrumentação analítica moderna, ajudando na popularização deste método e dos seus conceitos, pois permite atender aos rigorosos Limites Máximos de Resíduos, determinados por legislações internacionais [17].

Já para determinação desses resíduos em matrizes ambientais, análises cromatográficas gasosas e líquidas vêm sendo empregadas $[18,19]$. De modo geral, a cromatografia gasosa pode ser utilizada na análise de compostos voláteis e termicamente estáveis e a cromatografia líquida tem sido utilizada para determinação de compostos termicamente instáveis e não voláteis. Ambas as técnicas, são muito usadas na determinação de pesticidas em diferentes matrizes ambientais, como água, solo e sedimento [20, 21, 22, 23].

Neste contexto, a necessidade de identificar um método eficiente para determinar a concentração do PBZ presentes nos solos cultivados com diferentes variedades de mangueira, no Vale do Submédio São Francisco é fundamental. Uma vez que existe a necessidade de analisar os teores residuais desse composto antes de uma nova aplicação, com vistas a minimizar o dano ambiental e econômico na produção. 
Neste contexto, o objetivo desse estudo foi validar um método para quantificar paclobutrazol no solo, avaliando áreas cultivadas com diferentes variedades de mangueira no Vale do São Francisco.

\section{MATERIAL E MÉTODOS}

\subsection{Reagentes e soluções}

Padrões de paclobutrazol (PBZ) adquiridos da Sigma-Aldrich com pureza 99,6\%, metanol e acetonitrila todos grau HPLC da J. T. Baker, sulfato de magnésio anidro, cloreto de sódio, hidrogeno citrato dissódico sesqui-hidratado e citratotrissódicodi-hidratado foram adquiridos da J.T.Baker.

\subsection{Amostra}

Foram coletadas 30 amostras de solo em três fazendas produtoras de diferentes variedades de mangueira (Tommy Atkins- TA, Palmer-PA, Keitt-KT, Kent-KN), em Petrolina-PE, e híbridos (H), no campo experimental da Embrapa em Juazeiro-BA. As identificações das amostras são: testemunha (1 amostra de área de caatinga preservada com solo representativo dessas áreas), cultivados com as variedades de mangueira Tommy Atkins (5 amostras), Palmer (2 amostras), Keitt (4 amostras), Kent (11 amostras) e híbridos de mangueira (cruzamento com diferentes variedades) (8 amostras). Na Tabela 1, encontra-se o histórico dos solos coletados, as variedades de mangueira e data da aplicação do PBZ.

O solo foi coletado na profundidade de $0-20 \mathrm{~cm}$, onde inicialmente removeu-se a matéria orgânica livre no solo (restos vegetais oriundos da poda e adudos orgânicos) com uma espátula. As coletas foram entre a projeção da copa e o colo da planta, local de aplicação do PBZ e da água oriundo da irrigação. As amostras de solos foram coletadas em cerca de 10 plantas por área de um hectare. Em cada planta, coletaram-se 5 amostras simples com trado holandês e colocou-se em um balde para preparar a amostra composta. Ao final, coletaram-se 50 amostras simples para compor uma composta da área, em cada uma das variedades de mangueira estudada. No laboratório, as amostras foram colocadas em bandejas plásticas e postas para secar a temperatura ambiente. Foram realizadas análises física e química de solo segundo metodologia da Embrapa (1997) [24].

\subsection{Extração do PBZ do solo}

Foram testadas duas metodologias para extração do PBZ no solo: Ultrasom e QuEchErs. Inicialmente, um solo livre de contaminação coletado de uma área do campo experimental da Embrapa Semiárido foi fortificado com concentrações de 2 e $20 \mathrm{mg} \mathrm{kg}^{-1}$ de PBZ.

\section{Ultrassom}

Para a extração por ultrassom, foram pesados $2,5 \mathrm{~g}$ de solo e colocado em tubo de centrífuga com capacidade para $50 \mathrm{~mL}$, adicionado-se $30 \mathrm{~mL}$ de metanol grau HPLC, sendo a mistura levada ao ultrassom por 30 minutos, e em seguida a centrifugação por $3500 \mathrm{rpm}$ por 5 minutos. Esse mesmo procedimento foi realizado mais duas vezes. O sobrenadante foi recolhido no balão, rotaevaporado até a secura e ressuspendido em metanol, para posterior análise no HPLC. Esse ensaio foi realizado em quadruplicata para as duas contaminações do solo.

\section{QuEChERS}

O segundo método de extração usado foi o QuEChERS, pesando-se $10 \mathrm{~g}$ de solo, colocando-o em um tubo falcon de $50 \mathrm{~mL}$ e adicionando-se $10 \mathrm{~mL}$ de acetonitrila grau HPLC. A mistura foi submetida à vigorosa agitação no vortex por 1 minuto e em seguida, foi adicionado os sais de QuEChERS (1 g de citrato trissódico di-hidratado (TSCD) e 0,5g de hidrogeno citrato de dissódio sesqui-hidratado (DHS)). Foi realizada nova agitação, seguida de centrifugação a $3500 \mathrm{rpm}$ durante $5 \mathrm{~min}$. Para o clean up, 1,0 mL desse extrato foi transferido para outro tubo, que continha $150 \mathrm{mg}$ de sulfato de magnésio e $25 \mathrm{mg}$ PSA. Essa mistura foi novamente agitada por 30 segundos e centrifugada a $3500 \mathrm{rpm}$ por 5 minutos. O sobrenadante retirado foi filtrado (Syringe Filters Nylon $0,45 \mu \mathrm{m})$ e injetado no HPLC. Esse procedimento também foi realizado em quadruplicata. 
Tabela 1: Histórico da área de estudo dos solos coletados nas áreas cultivadas com diferentes variedades de mangueira na região do Vale do São Francisco

\begin{tabular}{lllc}
\hline \multicolumn{1}{c}{ Identificação } & Variedade cultivada & Data da última aplicação & Data da coleta \\
\hline TA1 & Testemunha & Sem histórico de PBZ & $07 / 2014$ \\
TA2 & Tommy Atkins & $11 / 2011$ & $12 / 2012$ \\
TA3 & Tommy Atkins & $12 / 2011$ & $12 / 2012$ \\
TA4 & Tommy Atkins & $01 / 2014$ & $05 / 2014$ \\
TA5 & Tommy Atkins & $01 / 2014$ & $05 / 2014$ \\
TA6 & Tommy Atkins & $01 / 2014$ & $05 / 2014$ \\
& & & \\
PA1 & Palmer & $11 / 2012$ & $12 / 2012$ \\
PA2 & Palmer & $08 / 2012$ & $12 / 2012$ \\
KN1 & Kent & $01 / 2014$ & $05 / 2014$ \\
KN2 & Kent & $01 / 2014$ & $07 / 2014$ \\
KN3 & Kent & $01 / 2014$ & $07 / 2014$ \\
KN4 & Kent & $01 / 2014$ & $07 / 2014$ \\
KN5 & Kent & $01 / 2014$ & $07 / 2014$ \\
KN6 & Kent & $01 / 2014$ & $07 / 2014$ \\
KN7 & Kent & $01 / 2014$ & $07 / 2014$ \\
KN8 & Kent & $01 / 2014$ & $07 / 2014$ \\
KN9 & Kent & $01 / 2014$ & $07 / 2014$ \\
KN10 & Kent & $07 / 2014$ & $11 / 2014$ \\
KN11 & Kent & $01 / 2014$ & $07 / 2014$ \\
KT1 & Keit & $12 / 2013$ & $07 / 2014$ \\
KT2 & Keit & $01 / 2014$ & $05 / 2014$ \\
KT3 & Keit & $11 / 2012$ & $12 / 2012$ \\
KT4 & Keit & $02 / 2014$ & $07 / 2014$ \\
H1 & Híbrido & $04 / 2014$ & $03 / 2015$ \\
H2 & Híbrido & $04 / 2014$ & $03 / 2015$ \\
H3 & Híbrido & $04 / 2014$ & $03 / 2015$ \\
H4 & Híbrido & $04 / 2014$ & $03 / 2015$ \\
H5 & Híbrido & $04 / 2014$ & $03 / 2015$ \\
H6 & Híbrido & $04 / 2014$ & $03 / 2015$ \\
H7 & Híbrido & $04 / 2014$ & $03 / 2015$ \\
\hline
\end{tabular}

\subsection{Identificação e quantificação do PBZ no solo- Condições cromatográficas}

O extrato com o PBZ foi analisado por cromatografia líquida (Alliance Walters $2695 \mathrm{com}$ detector UV/Vis Walters 2489), usando coluna C-18 (SunfireTM $5 \mu \mathrm{m}$ 4,6x150 mm), fase móvel $\mathrm{MeOH}: \mathrm{H}_{2} \mathrm{O}$, na proporção de 80:20, com fluxo de $1 \mathrm{~mL} \mathrm{~min}^{-1}$, volume de injeção de $10 \mu \mathrm{L}$, a temperatura do forno de $40^{\circ} \mathrm{C}$ e comprimento de onda de $220 \mathrm{~nm}$.

\subsection{Validação da metodologia}

Para a validação do método, foram analisados os seguintes parâmetros:

1) Seletividade - Foi avaliada por meio dos tempos de retenção do padrão de concentração de 100 $\mathrm{mg} \mathrm{L}{ }^{-1}$, branco e da amostra fortificada na concentração de $1 \mathrm{mg} \mathrm{Kg}^{-1}$.

2) Linearidade - Foi avaliada por meio da construção de uma curva de calibração do paclobutrazol em metanol $\left(0,2 ; 0,5 ; 1,0 ; 1,2 ; 2,0 ; 5,0 ; 10,0 ;\right.$ e $\left.20,0 \mathrm{mg} \mathrm{L}^{-1}\right)$. Cada ponto da curva foi injetado 5 vezes e os dados de regressão linear foram obtidos por meio do software do equipamento. $\mathrm{O}$ critério mínimo aceitável do coeficiente de correlação foi de 0,999 [22]. 
3) Limites de detecção e quantificação - Os limites de detecção (LD) e quantificação (LQ) foram calculados para o paclobutrazol pelas equações $\mathrm{LD}=3 \mathrm{~s} / \mathrm{m} \mathrm{e} \mathrm{LQ}=10 \mathrm{~s} / \mathrm{m}$, respectivamente, onde s é o desvio padrão da área da menor concentração da curva $\left(0,2 \mathrm{mg} \mathrm{L}^{-1}\right)$, injetados sete vezes, e $\mathrm{m}$ é o coeficiente angular da curva de calibração [22,24].

4) Exatidão - A exatidão do método foi avaliada por meio de ensaios de recuperação, fortificando com cinco concentrações $\left(1,0 ; 2,0 ; 5,0 ; 10,0 ;\right.$ e $\left.20,0 \mathrm{mg} \mathrm{Kg}^{-1}\right)$ um solo livre de contaminação. Essas amostras foram extraídas em triplicata por meio da extração QuEChErs. Os resultados obtidos foram expressos em termos de \% de recuperação.

5) Precisão - Para a determinação da precisão, foram realizados ensaios de repetibilidade e precisão intermediária.

5.1) Repetibilidade - A repetibilidade foi avaliada por meio da injeção em triplicata de amostras de solo fortificadas com paclobutrazol, nos níveis de 1,0;2,0; 5,0;10,0; e 20mg Kg ${ }^{-1}$, sendo expressa como \% do coeficiente de variação (CV).

5.2) Precisão intermediária - Para a verificação deste parâmetro, foram utilizadas as mesmas amostras já citadas na avaliação da repetibilidade, as quais foram injetadas em triplicata a cada três dias, perfazendo um total de 9 injeções para cada amostra. A precisão intermediária foi expressa como \% do coeficiente de variação (CV).

\section{RESULTADOS E DISCUSSÃO}

\subsection{Avaliação da extração: ultrassom e QuEchers}

Os resultados extração do paclobutrazol no solo pelo método do ultrassom e QuEChers encontram- se na Tabela 2.

Tabela 2: Comparação entre o método QuEChERS e ultrassom nos níveis 2 e $20 \mathrm{mg} \mathrm{Kg}^{-1} \mathrm{de} P B Z$ nas amostras de solo

\begin{tabular}{cccc}
\hline Método & Fortificação $\left(\mathrm{mg} \mathrm{Kg}^{-1}\right)$ & Recuperação $\left(\mathrm{m} \mathrm{L} \mathrm{L}^{-1}\right)$ & Recuperação $(\%)$ \\
\hline Ultrassom & 2 & $1,8 \pm 0,2$ & 90 \\
& 20 & $18,8 \pm 2,7$ & 94 \\
QuEChERS & 2 & $2,1 \pm 0,2$ & 105 \\
& 20 & $20,0 \pm 4,8$ & 100 \\
\hline
\end{tabular}

De acordo com os resultados de recuperação apresentados na Tabela 2, é possível verificar que os valores de recuperação para os dois métodos foram satisfatórios, pois apresentaram a recuperação maior ou igual a 90\%, dentro da faixa aceitável que é de 70-120\% [23]. Nesse contexto, para avaliação do PBZ dos solos cultivados com mangueira, fez-se a opção pelo método QUeCHERS, pelo seu baixo consumo de solvente e maior percentual de recuperação.

\subsection{Validação do método}

Seletividade

Na Figura 1, observando o cromatograma do branco da amostra, não se observa o sinal analítico do PBZ no tempo de retenção de 2,8 min, enquanto que no padrão e amostra apresenta o pico para esse tempo de retenção, evidenciando que o método é seletivo para esse composto. 


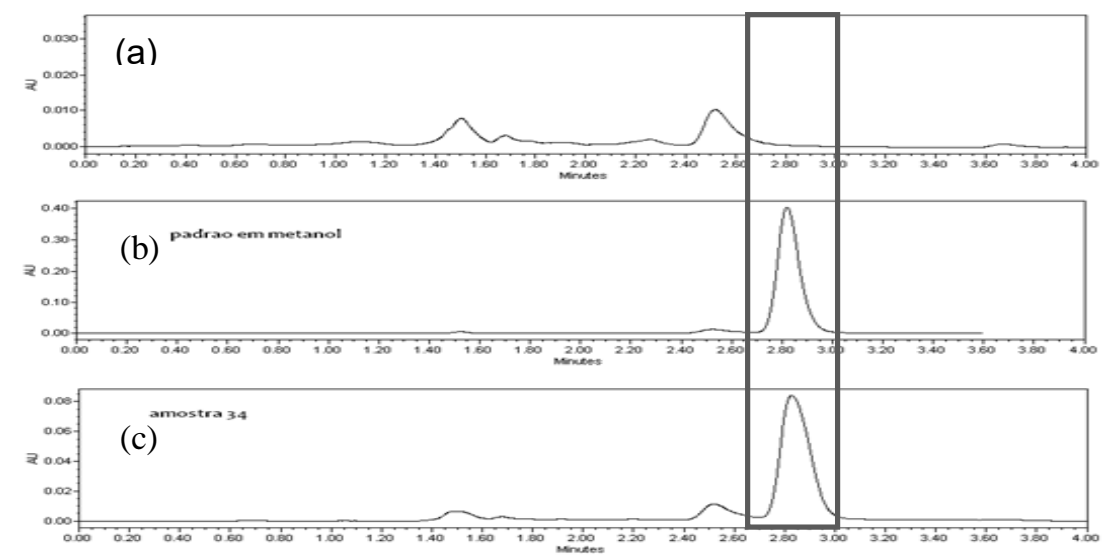

Figura 1: Cromatogramas branco da amostra (a), padrão de $100 \mathrm{mg} \mathrm{L}^{-1} \mathrm{PBZ}(\mathrm{b})$ e amostra fortificada com $1 \mathrm{mg} \mathrm{kg}^{-1} \mathrm{PBZ}(\mathrm{c})$

Linearidade, limite de detecção e quantificação do PBZ no solo

Na tabela 3, encontra-se a equação da reta, $\mathrm{R}^{2}$, limite de detecção e quantificação. A linearidade do método foi confirmada pelo $\mathrm{R}^{2}$ de 0,9996 na faixa de concentração estudada. O limite detecção foi $0,042 \mathrm{mg} \mathrm{kg}^{-1} \mathrm{e}$ o de quantificação $0,139 \mathrm{mg} \mathrm{kg}^{-1}$.

Tabela 3: Faixa linear, coeficiente de correlação $\left(R^{2}\right)$, equação da reta, limite de detecção $(L D) e$ quantificação $(L Q)$ do $P B Z$

\begin{tabular}{ccccc}
\hline $\begin{array}{c}\text { Faixa linear } \\
\mathrm{mg} \mathrm{L}^{-1}\end{array}$ & Equação da reta & $\mathrm{R}^{2}$ & $\mathrm{LD}$ & $\mathrm{LQ}$ \\
\hline $0,2-20$ & $21629 \mathrm{x}-1221,5$ & 0,9996 & 0,042 & 0,139 \\
\hline
\end{tabular}

Exatidão, repetibilidade e precisão intermediária

A exatidão foi verificada por meio dos percentuais de recuperação das amostras fortificadas. Na Tabela 4, pode-se observar que a recuperação variou de 101 a 107\%. Esses valores são aceitáveis de acordo com a literatura, segundo Ribani et al (2004) [23], cujos valores podem variar de 70$120 \%$. A precisão do método foi avaliada em função da repetibilidade e da precisão intermediária. Os valores de CV para a repetibilidade ficou na faixa de 1,16-3,16\% e precisão intermediária na faixa de 1,43-3,29\%. Os valores de CV para a repetibilidade e precisão intermediária ficaram muito próximos e menores que $20 \%$, indicando a adequada precisão do método. Segundo Ribani et al (2004) [23], é aceitável CV (\%) de até $20 \%$.

Tabela 4: Exatidão e precisão do método para quantificação de PBZ no solo

\begin{tabular}{cccc}
\hline $\begin{array}{c}\text { Nivel de fortificação } \\
\left(\mathrm{mg} \mathrm{kg}^{-1}\right)\end{array}$ & Exatidão (\%) & Repetibilidade (CV\%) & $\begin{array}{c}\text { Precisão intermediária } \\
(\mathrm{CV} \%)\end{array}$ \\
\hline 1 & 105 & 3,03 & 3,06 \\
2 & 107 & 3,16 & 3,29 \\
5 & 105 & 1,49 & 1,65 \\
10 & 107 & 1,16 & 1,50 \\
20 & 101 & 1,92 & 1,43 \\
\hline
\end{tabular}

\subsection{Caracterização do solo}

As características dos solos estudados encontram-se na tabela 5. Os solos estudados de origem Petrolina/PE e Juazeiro/BA apresentam características diferentes, principalmente em relação ao aspecto fisico. Os solos coletados das fazendas em Petrolina apresentam textura arenosa e mais pobre em matéria orgânica, enquanto o de Juazeiro de textura argilosa e mais rico em matéria 
orgânica. Ambos os solos apresentaram pH próximo a neutralidade, baixa condutividade e baixo teor de matéria orgânica.

Tabela 5: Caracterização fisica e química dos solos estudados

\begin{tabular}{ccccccc}
\hline Solo & $\begin{array}{c}\text { Areia } \\
\left(\mathrm{kg} \mathrm{dm}^{-3}\right)\end{array}$ & $\begin{array}{c}\text { Silte } \\
\left(\mathrm{kg} \mathrm{dm}^{-3}\right)\end{array}$ & $\begin{array}{c}\text { Argila } \\
\left(\mathrm{kg} \mathrm{dm}^{-3}\right)\end{array}$ & $\mathrm{pH}$ & $\begin{array}{c}\mathrm{CE} \\
\left(\mathrm{ms} \mathrm{cm}^{-1}\right)\end{array}$ & $\begin{array}{c}\mathrm{MO} \\
\left(\mathrm{g} \mathrm{kg}^{-1}\right)\end{array}$ \\
\hline $\begin{array}{c}\text { Petrolina } \\
\text { Min/max }\end{array}$ & $654,4-812,5$ & $101,1-212,6$ & $33,8-89,2$ & $6,1-7,2$ & $0,28-1,19$ & $6,7-14$ \\
\hline $\begin{array}{c}\text { Juazeiro } \\
\text { Min/max }\end{array}$ & $292,6-314,8$ & $289,8-351,7$ & $355,8-394,4$ & 7,5 & $0,35-0,36$ & $17,2-19,1$ \\
\hline
\end{tabular}

Min/max - Valores mínimos e máximos, CE - Condutividade elétrica e MO - Matéria orgânica

\subsection{Aplicação do método proposto}

Depois de validado o método, esse foi aplicado na determinação de PBZ em amostras de solo cultivado com diferentes variedades de mangueira (Tommy Atkins, Palmer, Kent e Keit) e hibridos do Vale do São Francisco. Na Tabela 6, encontra-se a concentração de PBZ das 30 amostras compostas de solos coletadas nas áreas de cultivo.

\begin{tabular}{|c|c|c|c|}
\hline Identificação & $\begin{array}{l}\text { Variedades } \\
\text { cultivadas }\end{array}$ & $\begin{array}{c}\text { Concentrações PBZ } \\
\left(\mathrm{mg} \mathrm{kg}^{-1}\right)\end{array}$ & *TAA (Dias) \\
\hline TA1 & Branco & $<\mathrm{Ld}$ & - \\
\hline TA2 & Tommy Atkins & $<\mathrm{Ld}$ & $>1000$ \\
\hline TA3 & Tommy Atkins & $<\mathrm{Ld}$ & $>1000$ \\
\hline TA4 & Tommy Atkins & 2,26 & 150 \\
\hline TA5 & Tommy Atkins & 1,17 & 150 \\
\hline TA6 & Tommy Atkins & $<\mathrm{Ld}$ & $>1000$ \\
\hline PA1 & Palmer & $<\mathrm{Ld}$ & $>700$ \\
\hline PA2 & Palmer & $<\mathrm{Ld}$ & $>700$ \\
\hline KN1 & Kent & $<\mathrm{Ld}$ & 150 \\
\hline KN2 & Kent & 3,63 & 150 \\
\hline KN3 & Kent & 3,18 & 150 \\
\hline KN4 & Kent & 6,86 & 150 \\
\hline KN5 & Kent & 4,59 & 150 \\
\hline KN6 & Kent & 4,72 & 150 \\
\hline KN7 & Kent & 4,74 & 150 \\
\hline KN8 & Kent & 5,93 & 150 \\
\hline KN9 & Kent & 3,52 & 150 \\
\hline KN10 & Kent & $<\mathrm{Ld}$ & $>700$ \\
\hline KN11 & Kent & 0,14 & 150 \\
\hline KT1 & Keit & $<\mathrm{Ld}$ & $>700$ \\
\hline KT2 & Keit & 1,49 & 150 \\
\hline KT3 & Keit & 0,18 & 150 \\
\hline KT4 & Keit & $<\mathrm{Ld}$ & $>700$ \\
\hline H1 & Híbrido & 0,74 & 330 \\
\hline $\mathrm{H} 2$ & Híbrido & 1,28 & 330 \\
\hline $\mathrm{H} 3$ & Híbrido & 3,73 & 330 \\
\hline $\mathrm{H} 4$ & Híbrido & 0,89 & 330 \\
\hline H5 & Híbrido & 1,78 & 330 \\
\hline H6 & Híbrido & 0,25 & 330 \\
\hline $\mathrm{H} 7$ & Híbrido & 0,62 & 330 \\
\hline
\end{tabular}

*TAA = tempo decorrido entre a aplicação de PBZ e a amostragens dos solos 
Ld= Limite de detecção

O método proposto foi capaz de quantificar PBZ nos solos, cujas concentraçãões variaram de 0,14 a $6,86 \mathrm{mg} \mathrm{kg}^{-1}$. Dentre as áreas estudadas, a maior concentração de PBZ foi encontrado nos solos cultivados com a variedade Kent, aos 150 dias após sua aplicação. Sabe-se que o manejo da produção na 'Kent', dado as suas características de maior vigor da cultivar, demanda maiores quantidades do regulador vegetal de crescimento (PBZ) para a indução ao repouso dos ramos [26]. Assim já eram esperados maiores teores de resíduos de PBZ nos solos cultivados com esta variedade, o qual vem a acarretar o aumento do risco de contaminação ambiental. Um dos produtores consultados revelou que vem aplicando nessa variedade o triplo da dose em relação às demais.

Em relação as áreas do cultivo dos híbridos, observa-se a presença de PBZ após 300 dias de aplicação. Isto se deve a característica do solo de natureza argilosa, que consegue reter por mais tempo esse composto. Pode-se observar também na Tabela 6, que com aproximadamente 5 meses após aplicação ainda é encontrada a presença desse resíduo no solo, sendo ideal, a cada aplicação do PBZ, o produtor realizar uma análise do solo antes para verificar a existência do PBZ no solo.

Já para outras amostras de solo com mais de 700 dias de aplicação de PBZ, a concentração ficou abaixo do $\mathrm{LD}\left(0,042 \mathrm{mg} \mathrm{kg}^{-1}\right)$, como pode ser observado na Tabela 6 . Isso pode ter acontecido devido a lixiviação do composto do solo, pois em muitas dessas áreas estudadas, o solo é arenoso com baixo teor de matéria orgânica (Tabela 5), ou também pelo processo de degradação, apesar da persistência desses compostos. Ferracini et al (2008) [11] avaliou a concentração de PBZ em 24 amostras de solos de duas fazendas com cultivo de mangueira no Vale do São Francisco, e observou que a concentração variou de 0,02 a $1,43 \mathrm{mg} \mathrm{kg}^{-1}$, embora no trabalho não cita a variedade da mangueira, enquanto nesse estudo variou de 0,14 a $7,0 \mathrm{mg} \mathrm{kg}^{-1}$. Essa variação deve-se principalmente a variedade Kent, pois nessa cultivar de mangueira se utiliza maior dosagem de PBZ também.

Sharma e Awasthi (2005) [8] verificaram a presença de PBZ no solo após 240 dias de aplicação. Outros estudos demonstraram a persistência do PBZ em até 3 anos após a aplicação [26, 27].

\section{CONCLUSÃO}

A extração do PBZ no solo por QuEchErs e a sua determinação por cromatografia líquida foi capaz de avaliar a concentração desse composto no solo em áreas que receberam doses regulares. Os parâmetros de validação avaliados evidenciaram que o método é preciso e exato. Foram quantificadas concentrações de PBZ em solos cultivados com diferentes variedades de mangueira, merecendo destaque os solos cultivados com a variedade Kent, pois apresentaram as maiores concentrações, servindo de alerta e atenção para os produtores, principalmente com relações aos possíveis problemas relacionados à poluição ambiental.

\section{AGRADECIMENTOS}

Os autores agradecem à FUNARBE e a FACEPE.

\section{REFERÊNCIAS BIBLIOGRÁFICAS}

1. Cardoso CDL, Souza JS. Fruticultura tropical: perspectivas e tendências. REN. 2000 Jan-Mar;31(1):8495.

2. Anuário Brasileiro da Fruticultura - Brazilian Fruit Yearbook. Editora Gazeta; 2016.

3. Fonseca N, Neto MTC, Ledo CAS. Paclobutrazol no Florescimento e na Produção da Mangueira “TOMMY ATKINS". Ciênc Agrotec. 2004;28(1):807-14, doi:10.1590/S1413-70542004000400012.

4. Singh DK. Triazole Compounds in Horticulture. New Delhi: Agrotech Publishing Academy; 2001.

5. Davenport, T L. Reproductive physiology of mango. Brazilian Journal of Plant Physiology. 2007;19(4):363-376, doi:http://dx.doi.org/10.1590/S1677-04202007000400007

6. MOUCO, M. A. do C. (Ed.). Cultivo de mangueira. 3. Ed. Petrolina: Embrapa Semiárido; 2015 (Embrapa Semiárido. Sistemas de Produção, 3). 
7. Rademacher W, Spinelli F, Costa G. Prohexadione-Ca: Modes of action of a multifuncional plant bioregulator for fruit trees. Act Hort. 2006;727: 97-106, doi:10.17660/ActaHortic.2006.727.10.

8. Sharma D, Awasthi MD. Uptake of soil applied paclobutrazol in mango (Mangifera indica L.) and its persistence in fruit and soil. Chemosphere. 2005;60(2):164-9, doi:10.1016/j.chemosphere.2004.12.069.

9. Silva CMMS, Fay EF, Jonsson CM. Impacto ambiental do regulador de crescimento vegetal paclobutrazol. Brasília : Embrapa Meio Ambiente; 2003. 106p. (Documento, 30).

10. Mouco, MAdo C, Ono EO, Rodrigues JD. Controle do crescimento vegetativo e floração de mangueira Kent. Rev Bras Frutic. 2011;33(4):1043-47, doi:10.1590/S0100-29452011000400001.

11. Ferracini VL, Queiroz SC, Gomes MAF, Rosa MA. Determinação de paclobutrazol em solo por cromatografia liquida. Brasília: Embrapa Meio Ambiente; 2008. 4p. (Comunicado técnico, 49).

12. Prestes OD, Adaime MB, Zanella R. QuEChERS: possibilidades e tendências no preparo de amostra para determinação multirresíduo de pesticidas em alimentos. Scientia Chromatographica. 2011;3(1):51-64, doi:10.4322/sc.2011.004.

13. Prestes OD, Friggi CA, Adaime MB, Zanella R. Quechers - um método moderno de preparo de amostra para determinação multirresíduo de pesticidas em alimentos por métodos cromatográficos acoplados à espectrometria de massas. Quim Nova. 32(6):1620-34, doi: 10.1590/S0100-40422009000600046.

14. Bragança I, Plácido A, Paíga P, Domingues VF, Delerue-Matos C. QuEChERS: a new sample preparation approach for the determination of ibuprofen and its metabolites in soils. Sci Total Environ. 2012 Set;433:281-9, doi:10.1016/j.scitotenv.2012.06.035.

15. Martins GL. Determinação de resíduos de pesticidas em solo de lavoura de arroz irrigado empregando quechers modificado e LC-Ms/Ms[dissertation]. Santa Maria (BR): Universidade Federal de Santa Maria; 2010.149p.

16. Prestes OD, Martins ML, Friggi CA, Munaretto JS, Adaime MB, Zanella R. O estado da arte na determinação de resíduos de medicamentos veterinários em alimentos de origem animal empregando técnicas cromatográficas acopladas à espectrometria de massas. Quim Nova. 2013;36(5):697-710, doi:10.1590/S0100-40422013000500015.

17. Galli A, De Souza D, Garbellini GS, Coutinho CFB, Mazo LH, Avaca LA, Machado SAS. Utilização de técnicas eletroanalíticas na determinação de pesticidas em alimentos. Quím Nova. 2006;29(1):105-12, doi:10.1590/S0100-40422006000100020.

18. Chiaradia MC, Collins CH, Jardim CSF. O estado da arte da cromatografia associada à espectrometria de massas acoplada à espetrometria de massas na análise de compostos tóxicos em alimentos. Quím Nova. 2008;31(3):623-36, doi:10.1590/S0100-40422008000300030.

19. Pinho GP, Neves AA, Queiroz MELR, Silvério FO. Efeito de matriz na quantificação de agrotóxicos por cromatografia gasosa. Quím Nova. 2009;32(4):987-95, doi:10.1590/S0100-40422009000400030.

20. Silva JCC. Desenvolvimento de metodologia analítica para determinação de microcistina-LR e agrotóxico em águas superficiais utilizando as técnicas de cromatografia líquida e cromatografia gasosa acopladas a espectrometria de massas[dissertação]. Outro Preto (BR): Universidade de Ouro Preto; 2010. 176p.

21. Pastor-Belda M, Garrido I, Campillo N, Vinas P, Hellin P, Flores P, Fenol J. Determination of spirocyclic tetronic/tetramic acid derivatives and neonicotinoid insecticides in fruits and vegetables by liquid chromatography and mass spectrometry after dispersive liquid-liquid microextraction. Food Chem. 2016 Jul;202:389-95, doi:10.1016/j.foodchem.2016.01.143.

22. Caldas SS, Rombaldi C, Arias JLO, Marube LC, Primel EG. Multi-residue method for determination of 58 pesticides, pharmaceuticals and personal care products in water using solvent demulsification dispersive liquid-liquid microextraction combined with liquid chromatography-tandem mass spectrometry. Talanta. 2016 Jan;146(1):676-88, doi:10.1016/j.talanta.2015.06.047.

23. Ribani M, Bottoli CBG, Collins CH, Jardim ICSF, Melo LFC. Validação em métodos cromatográficos e eletroforéticos. Quim Nova. 2004; 27(5):771-780, doi:10.1590/S0100-40422004000500017.

24. Embrapa. Manual de métodos de análise de solo. Brasília: Embrapa; 1997.

25. Harris DC. Análise química quantitativa. Rio de Janeiro: LTC; 2008.

26. Jacyna T, Dodds KG. Some effects of soil-applied paclobutrazol on performance of 'Sundrop' apricot (Prunus armeniaca L) trees and on residue in the soil. New Zealand Journal of Crop and Horticultural Science 1995;23(3):323-29, doi:10.1080/01140671.1995.9513905.

27. Jacyna T, Dodds K G. Effect of method of application of paclobutrazol in high-density sweet cherry orchards on tree performance and apparent soil residue. Journal of Horticultural Science and Biotechnology. 1999;74(2):213-18, doi: 10.1080/14620316.1999.11511097. 